war veterans with/without post-traumatic stress disorder (PTSD). METHODS/STUDY POPULATION: The peripheral blood mononuclear cells (PBMCs) from war veterans with/without post-traumatic stress disorder (PTSD) were used to monitor transcription profile of the molecular markers of mitochondrial biogenesis and fusion/architecture, as well as the markers of mtDNA copy numbers. The human male immortalized monocytes were exposed in vitro to hormonal markers of PTSD in order to monitor the effects of each particular hormonal marker on the molecular markers of mitochondrial biogenesis and fusion/architecture, as well as the markers of mtDNA copy numbers. RQ-PCR analyses were used to define transcriptional profile of above mentioned markers. RESULTS/ ANTICIPATED RESULTS: The transcription profiles of above mentioned markers were disturbed, with high individual variability within the groups. A significant increase in the expression of the PPARGC1A transcript was observed in a group of subjects with current PTSD, as well as in the subjects with "life-time" PTSD, compared to healthy controls. PPARGC1B, NRF2 and MFN2 transcripts increased only in PBMCs of "life-time"-PTSD, while the level of transcripts for other investigated genes and the ratio of markers of mtDNA copy numbers showed no significant difference between groups. The in vitro results showed parallelism with the results obtained using the PBMCs from the subjects of the PTSD study. DISCUSSION/SIGNIFICANCE OF IMPACT: Although preliminary (the analysis require a larger number of subjects), the results are first findings and a solid base for further extensive multidisciplinary research in order to clarify the molecular mechanisms for the prevention and treatment of trauma-induced pathological conditions.

4564

Nilotinib alters microRNAs that regulate specific autophagy and ubiquitination genes in the cerebrospinal fluid of Parkinson's patients ${ }^{\dagger}$

Alan Fowler ${ }^{1}$, Yasar Torres-Yhagi ${ }^{2}$, Fernando Pagan², Michaeline Hebron $^{3}$, Barbara Willmarth ${ }^{2}$, Joy Arellano ${ }^{2}$, Helen Howard ${ }^{2}$, Sara Matar $^{3}$, Timothy Chiu ${ }^{3}$, Jaeil Ahn ${ }^{3}$, and Charbel Moussa ${ }^{3}$ ${ }^{1}$ Georgetown - Howard Universities; ${ }^{2}$ MedStar Georgetown University Hospital; ${ }^{3}$ Georgetown University Medical Center

OBJECTIVES/GOALS: Our preclinical data demonstrate that the principal effects of nilotinib, a multi-tyrosine kinase inhibitor, in models of neurodegeneration is clearance of misfolded proteins via autophagy. Here we aimed to evaluate the effects of nilotinib on microRNAs in the cerebrospinal fluid of Parkinson's disease patients. METHODS/ STUDY POPULATION: Cerebrospinal fluid (CSF) was collected as part of an open label phase I (NCT02281474) $(n=12,300 \mathrm{mg}$ nilotinib taken orally once daily for 6 months), and a phase II randomized, double-blind, placebo-controlled study (NCT02954978) ( $\mathrm{n}=75$, randomized 1:1:1 into placebo, $150 \mathrm{mg}$ or $300 \mathrm{mg}$ nilotinib taken orally once daily for 12 months). RNA was isolated from CSF and Indexed sequencing libraries were prepared from total RNA plus miRNA. Next generation whole-genome sequencing (single-end 1x75 bp, 25 million raw reads per sample) was performed to identify miRNAs significantly differentially expressed (fold-change $\geq 2$, BenjaminiHochberg FDR p-value $\leq 0.05$ or Empirical Bayes FDR $\leq 0.05$ ) with treatment compared to baseline. RESULTS/ANTICIPATED RESULTS: Next generation whole-genome sequencing of microRNAs in the CSF demonstrated that nilotinib significantly increases microRNAs that specifically regulate expression of autophagy and ubiquitination genes in individuals with Parkinson's disease. In the open label phase I, samples, 28 microRNAs found to regulate autophagy and ubiquitination genes, were significantly altered with treatment (Benjamini-Hochberg FDR p-value $\leq 0.05$ ). In the phase II randomized, double-blind, placebo-controlled study samples, we verified several of those 28 candidate microRNAs had been significantly deferentially expressed with treatment (Empirical Bayes FDR p-value $\leq 0.05)$. DISCUSSION/SIGNIFICANCE OF IMPACT: Our data provide robust evidence that nilotinib's effects on misfolded protein clearance is via autophagy and CSF miRNA sequencing is a valid biomarker of nilotinib's effects in a definitive phase III study to investigate nilotinib in Parkinson's and other neurodegenerative diseases. CONFLICT OF INTEREST DESCRIPTION: Charbel Moussa is listed as an inventor on several Georgetown University patents for the use of tyrosine kinase inhibitors as a treatment for neurodegenerative diseases

\section{Periodontal disease and the oral microbiome in antiretroviral-treated patients with HIV}

Medini K Annavajhala ${ }^{1}$, Jayesh G. Shah ${ }^{2}$, Jessica Weidler ${ }^{2}$, Karolina Kister $^{2}$, Ryan T. Demmer ${ }^{3}$, Sunil Wadhwa², Michael T. Yin ${ }^{2}$, and Anne-Catrin Uhlemann ${ }^{2}$

${ }^{1}$ Columbia University, Irving Institute for Clinical; ${ }^{2}$ Columbia University Irving Medical Center; ${ }^{3}$ University of Minnesota

OBJECTIVES/GOALS: People living with HIV, despite antiretroviral therapy (ART), have increased burden of inflammatory and aging-related comorbidities such as periodontitis. Oral microbiota have been linked to periodontitis, but not in the context of HIV. We aim to compare relationships between the oral microbiome and periodontal disease in $\mathrm{HIV}+$ vs healthy controls. METHODS/STUDY POPULATION: In an ongoing cohort study we have been recruiting pre- and post-menopausal women with $\mathrm{HIV}+$ on ART for $\geq 6$ months and HIV- controls matched by menopausal status (target $n=30$ per arm; currently HIV $+: n=30$ postand 9 pre-M; HIV-: $\mathrm{n}=15$ post- and 6 pre-M). Patients age $<18$ or on antibiotics within 3 mos., except prophylaxis, are excluded. Patients provide saliva, then subgingival plaque collection during a dental examination through scaling from six index teeth. Standard CDC/AAP classifications of periodontitis are used. We will perform 16S rRNA and ITS sequencing to profile bacterial and fungal communities in saliva and plaque. Linear mixed effect regression and differential abundance analyses will be used to identify microbial and mycobial oral signatures of periodontal disease severity in HIV+ and HIV- populations. RESULTS/ANTICIPATED RESULTS: We found a markedly high prevalence of severe periodontal disease in HIV + women despite ART (59\%, compared to $11 \%$ in HIV-controls). In post-menopausal women with HIV, saliva bacterial $\alpha-$ and $\beta$-diversity in the saliva differed significantly with periodontal disease severity. Fungal $\alpha$-diversity was also significantly lower in plaque from teeth with severe loss of tissue attachment (CAL $\geq 4$ $\mathrm{mm}$ ). We identified bacterial and fungal taxa significantly enriched in post-menopausal HIV + women with severe compared to no or mild periodontitis. We hypothesize, similarly, associations between the oral microbiome and periodontitis in HIV-controls. However, we expect overall diversity metrics to be significantly altered in $\mathrm{HIV}+$ compared to HIV- patients, indicating long-term dysbiosis despite treatment with ART. DISCUSSION/SIGNIFICANCE OF IMPACT: Contrasting associations between the oral microbiome and periodontal disease with respect to HIV will provide evidence for the role of microbiota in accelerated aging phenotype caused by HIV. Our results would also provide rationale for interventions 